\title{
Etudes ethnobotanique et écologique des plantes d'hygiène intime féminine utilisées à Kinshasa (République Démocratique du Congo)
}

\author{
N.O. KABENA ${ }^{1 *}$, K.N. NGOMBE ${ }^{2}$, K.N. NGBOLUA ${ }^{1}$, B.A. KIKUFI ${ }^{1}$, L. LASSA ${ }^{1}$, \\ E. MBOLOKO ${ }^{3}$, P.T. MPIANA ${ }^{4}$ et L.F. LUKOKI ${ }^{1}$ \\ ${ }^{I}$ Département de Biologie, Faculté des Sciences, BP 190 Kinshasa XI, Université de Kinshasa, RD Congo. \\ ${ }^{2}$ Centre d'Etude des Substances Naturelles d'Origine Végétale, Faculté des Sciences Pharmaceutiques, \\ BP 212 Kinshasa XI, Université de Kinshasa, RD Congo. \\ ${ }^{3}$ Département de Gynécologie et Obstétrique, Faculté de Médecine, BP 123 Kinshasa XI, \\ Université de Kinshasa, RD Congo. \\ ${ }^{4}$ Département de Chimie, Faculté des Sciences, BP 190 Kinshasa XI, Université de Kinshasa, RD Congo. \\ *Auteur correspondant ; E-mail: odettekabena@gmail.com; Tél. : +(243)816904370
}

\section{RESUME}

En Afrique, plus de $80 \%$ de la population recourent à la médecine traditionnelle en utilisant les espèces végétales pour résoudre le problème de santé primaire. Le but de ce travail a été d'identifier les plantes utilisées par des femmes à Kinshasa/République Démocratique du Congo, pour les soins intimes et de déterminer leurs caractéristiques écologiques. L'enquête ethnobotanique a concerné trois cents personnes comprenant cinquante huit herboristes, habitant Kinshasa. Trente sept espèces, regroupées en vingt deux familles botaniques, ont été identifiées comme plantes pour le soin de la région génitale. Les espèces de Rubiaceae étaient les plantes les plus citées. Au sujet de statut écologique, $32 \%$ d'espèces identifiées sont des microphanerophytes, $35 \%$ sont des espèces Afro-tropicales et $51 \%$ d'espèces sont de la savane. La feuille est la partie la plus utilisée $(65 \%)$ et les raisons à caractère sanitaire expliquent $54 \%$ de cas d'utilisation des plantes tandis que les raisons à caractère érotique comptent pour $46 \%$. Ces divers résultats laissent découvrir la richesse culturelle et traditionnelle que les plantes d'hygiène intime constituent. Elles peuvent servir de matériel pour la production des substances bioactives.

(C) 2014 International Formulae Group. All rights reserved.

Mots clés : Inventaire, plantes, région vaginale, santé primaire, erotique.

\section{INTRODUCTION}

En Afrique, plus de $80 \%$ de la population recourent à la médecine traditionnelle en utilisant les espèces végétales pour résoudre le problème de santé primaire (WHO, 2002). L'utilisation des plantes médicinales pour divers problèmes de santé à travers le monde serait soutenue par le choix, mais aussi du fait de la pauvreté grandissante des populations qui n'ont pas accès aux médicaments modernes à cause de leurs coûts très onéreux (Moroh et al., 2008). Parmi les préparations de plantes qui soigneraient diverses affections, il existe celles qui sont spécifiques aux femmes. Ces dernières sont conviées à utiliser certaines recettes pour la 
toilette de la partie génitale (Gafos et al., 2010). D’après Tinde et al. (2008), d'autres raisons qui justifient cette pratique sont que les hommes préfèreraient pénétrer un vagin non-dilaté pour avoir des frictions beaucoup plus grandes, que les fluides vaginaux seraient impurs et diminueraient la fertilité, qu'un sexe féminin humide serait synonyme d'infidélité.

Pourtant, ces pertes vaginales sont d'origine physiologique, mais dans certains cas peuvent être le signe d'une infection génitale. Les leucorrhées constituent un des motifs de consultation les plus fréquents en gynécologie et on estime que, chaque année, un milliard de femmes sont atteintes par une infection urinaire ou une infection génitale basse (Reid, 2002 ; Bohbot, 2008 ; Bohbot et Lepargneur, 2011). Cependant, suite à la pudeur et la pauvreté qui caractérisent les femmes africaines, beaucoup d'entre elles recourent à l'automédication ou à l'usage des plantes locales dans le but de soulager leurs maux.

La République Démocratique du Congo possède un vaste territoire où se retrouvent des écosystèmes variés et riches en diversité biologique. Ses ressources forestières couvrent environ $47 \%$ des forêts africaines (FAO, 2009). Selon Ngbolua (2012), presque toutes les populations du Congo dépendent des plantes médicinales pour leurs besoins de santé parce que les coûts de drogues conventionnelles sont souvent exorbitants. Cependant, il n'y a que peu de plantes médicinales dont l'innocuité a été démontrée. De toutes les espèces dénombrées par la banque des données NAPRALET, dont la grande partie se retrouve dans les régions tropicales et subtropicales, 58\% n'ont pas fait l'objet d'études biologiques et chimiques (Ngombe, 2010).

La présente étude s'inscrit dans le cadre de la valorisation des plantes locales et l'analyse de leur importance relative en vue d'une gestion durable de ces taxons végétaux et de leurs écosystèmes. Dans le souci de bénéficier du savoir ancestral, l'objectif de ce travail a été de dresser un listing des plantes utilisées en hygiène intime de la femme à Kinshasa.

\section{MATERIEL ET METHODES}

\section{Etudes ethnobotaniques \\ Enquête}

Nous avons interviewé, sur base de leur consentement, les femmes (en associations féminines, à l'église, dans les entreprises, les institutions d'enseignement, les centres hospitaliers, les marchés, ...) ainsi que les vendeurs des plantes médicinales dans six principaux marchés de Kinshasa (Marché central, Matete, Gambela, Liberté, Delvaux et Mbanza Lemba) (Figure 1).

L'enquête a couvert la période allant de janvier à décembre 2012. Elle avait pour but de recenser les plantes dont les recettes sont appliquées à la région vaginale. Les informations recueillies ont été en rapport avec les noms de la plante, son mode de préparation et d'administration ainsi que le but de son usage.

\section{Identification des espèces végétales}

L'ensemble des plantes a été identifié et conservé à l'Herbarium du Laboratoire de Botanique Systématique et d'Ecologie Végétale du Département de Biologie de l'Université de Kinshasa sous l'appellation : « collection Kabena 2013 » reprenant 37 espèces. Les noms scientifiques des espèces et des familles sont inspirés de Kikufi (2012) et de la Base de données des plantes d'Afrique (version 3.4.0).

\section{Etude écologique des plantes}

Les données ethnobotaniques recueillies ont ensuite été complétées par des informations obtenues des différents travaux de la littérature (Flore du Congo belge et du Ruanda-Urundi, 1948-1960; 1962-1963, 1967-1971; Flore d'Afrique centrale: ZaïreRwanda-Burundi, 1972-1992) pour classifier les types écologiques. 


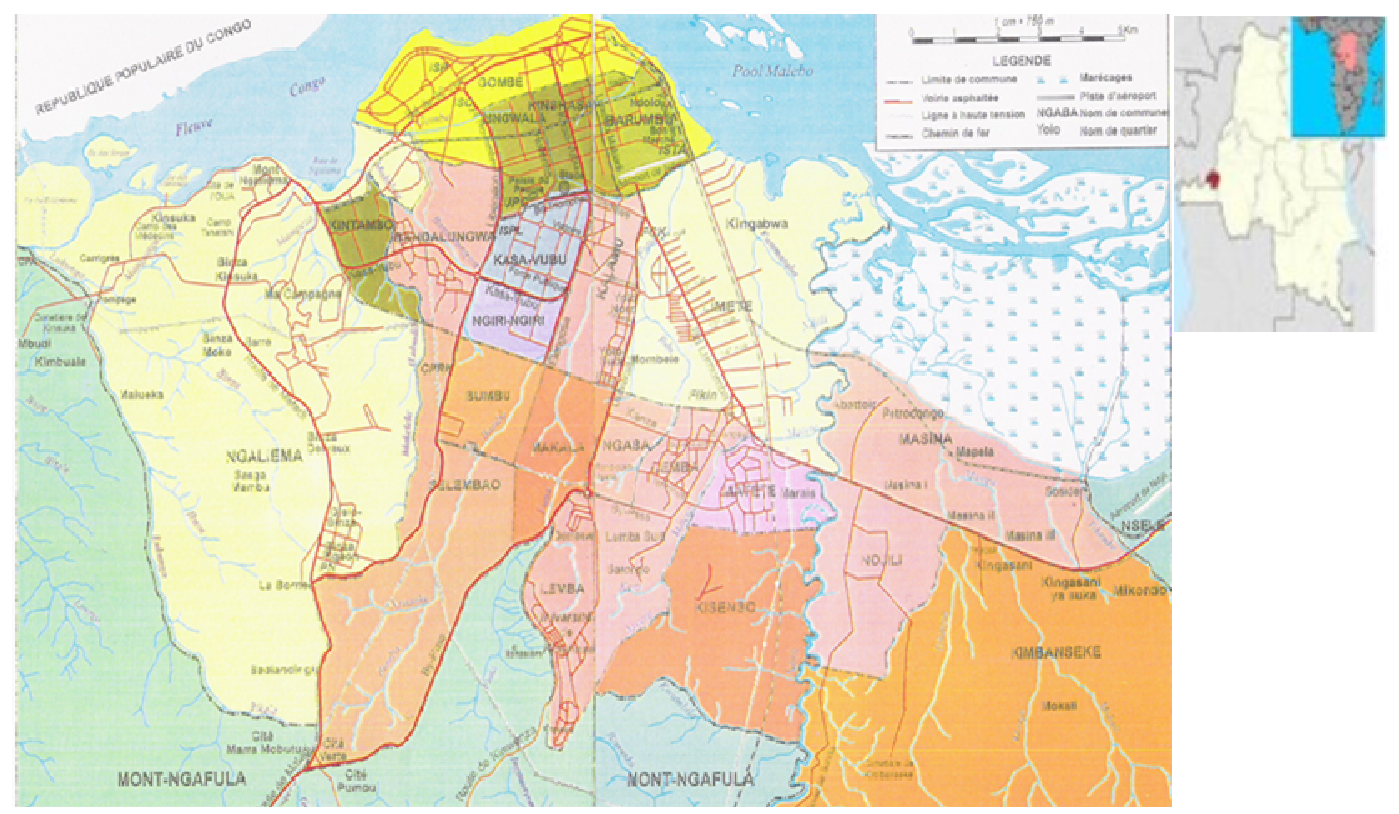

Figure 1 : Carte de la ville de Kinshasa. Source : Saint Moulin et Kalombo (2005).

\section{RESULTATS}

\section{Sujets interviewés}

L'enquête ethnobotanique a porté sur un total de 300 personnes dont 242 femmes et 58 vendeurs des plantes médicinales, parmi lesquels 35 hommes et 23 femmes (Figure 2).

De toutes les personnes ayant participé à l'étude, $7,7 \%$ d'entre elles seulement ont reconnu qu'elles utilisent les recettes à base de plantes en hygiène intime (Figure 3).

Les utilisatrices sont soit trouvées dans la catégorie des «vendeurs des plantes médicinales» ou dans celle des «femmes».

\section{Répertoire des plantes d'hygiène intime utilisées à Kinshasa}

Le Tableau 1 ci-dessous donne la liste des plantes d'hygiène intime, le nombre de fois cité, les parties exploitées ainsi que le but de leur utilisation. A côté de chaque espèce sont placées ses caractéristiques écologiques [Type morphologique : Arbre (A), Arbrisseau (ss/arb), Arbuste (arb), Herbe annuelle (Ha), Herbe vivace (Hv), Liane (Li) ou Sous-arbuste
(S/arb); Type biologique: Géophyte rhizomateux (Grh), Géophyte tubéreux (Gtu), Mésophanérophyte (MsPh), Microphanérophyte (McPh), Nanophanérophyte (Nph), Phanérophyte grimpant (Phgr), Thérophyte dressé (Thd) ou Thérophyte prostré (Thp); Type foliaire: Mégaphylle (Méga), Mésophylle (Méso) Microphylle (Micro) ou Nanophylle (Nano); Type de diaspores: Ballochores (Bal), Desmochores (Desmo), Sarcochores (Sarco), Sclérochores (Scl), Pogonochores (Pogo) ou Ptérochores (Ptéro); Distribution phytogéographique: Afroamericaine (AA), Afro-tropicale (AT), Congolaise (C), Cosmopolite (Cosm), Guinéenne (G), Guinéo-Congolaise (GC), Guinéo-Congolais-Zambézienne (GC-Z), Paléotropicale (Paléo), Pantropicale (Pan) et Type de biotope: Culture (Cult), Forêt primaire (FP), Forêt Secondaire (FS), Jachère (Jach), Savane (Sav) ou Rudérale (Rud)].

Il ressort du Tableau 1 que les 37 espèces inventoriées appartiennent à 22 familles botaniques. Sur ces 22 familles, la 
famille des Rubiaceae renferme cinq espèces tandis que 16 familles sont représentées par une espèce. La Figure 4 donne l'importance relative de ces 22 familles.

Il ressort de cette figure que les plantes appartenant à la famille des Rubiaceae ont été les plus citées.

\section{Connaissances sur l'usage des plantes}

Les plantes inventoriées peuvent être reparties en trois groupes en fonction des détenteurs de la connaissance sur leur usage en hygiène intime: les plantes connues par les femmes, celles citées uniquement par les vendeurs et enfin les plantes citées à la fois par les femmes et les vendeurs (Figure 5).

Il ressort de cette figure qu'environ $87 \%$ des plantes recensées sont connues par les vendeurs des plantes médicinales.

\section{Fréquence de citations des plantes inventoriées}

Les plantes qui ont été citées à plus de $50 \%$ se retrouvent dans la catégorie «citées à la fois par les femmes et les vendeurs». Il s'agit, dans l'ordre décroissant, des espèces suivantes: Alchornea cordifolia, Citrus limon, Zingiber officinale, Mangifera indica, Hibiscus mechowii, Abrus precatorius et Crossopteryx febrifuga. La Figure 6 donne la fréquence de citation de ces plantes en pourcentage.

Il ressort de cette figure qu'Alchornea cordifolia est la plante la plus citée de toutes. Elle a été mentionnée par 237 personnes sur les 300 interrogées.

\section{Partie utilisée pour composer les recettes}

Les résultats de l'enquête révèlent que différentes parties de plantes sont utilisées : feuille, écorce de tronc ou de racine, racine, rhizome, fruit et graine, follicules et les nodosités de racine. La feuille constitue l'organe qui compose la plupart des recettes inventoriées, elle en compose près de $65 \%$ (Figure 7).

\section{Buts de l'usage}

Treize (13) buts d'usage soutenant l'hygiène intime au moyen des recettes de plantes ont été déclarés par les femmes et les vendeurs de plantes médicinales. La Figure 8 ci-dessous présente les différents buts d'utilisation des plantes en hygiène intime et le nombre de fois qu'ils ont été répétés.

L'analyse de ces observations montre que le rétrécissement vaginal, la réduction des pertes vaginales, l'arrêt des lochies ainsi que la tonification des muscles vaginaux sont les raisons d'usage de plantes en hygiène intime qui sont les plus connues par les personnes interrogées. Les treize buts d'usage déclarés par les personnes interrogées peuvent être catégorisés en deux types (Figure 9) : l'usage d'ordre sanitaire où sont regroupées les raisons hygiéniques et thérapeutiques (7 raisons sur 13, soit 54\%) ainsi que l'usage d'ordre érotique (6 raisons sur 13, soit 46\%).

\section{Caractéristiques écologiques de plantes inventoriées}

Les plantes d'hygiène intime sont de 6 types morphologiques et le type arbuste est le plus représenté de ces plantes (soit 35\%) (Figure 10).

Les caractéristiques écologiques de plantes d'hygiène intime montrent également que les 37 espèces se repartissent en 10 types biologiques (Figure 11).

Les microphanérophytes couvrent la majorité des plantes d'hygiène intime, ils représentent $32 \%$. Les groupes de chaméphytes dressées, mégagéophytes et thérophytes prostrés représentent $3 \%$ chacun.

En ce qui concerne la distribution de types foliaires chez les plantes inventoriées (Figure 12), on peut remarquer que le type mésophylle est représenté à environ $43 \%$, alors que le type mégaphylle ne se trouve que chez une espèce végétale. 
Six (6) types de diaspores sont présents sur les plantes d'hygiène intime de la femme (Figure 13).

La répartition des types de diaspores de plantes d'hygiène intime montre que les sarcochores se retrouvent chez 19 espèces d'hygiène intime sur les 37 recensées, soit $51 \%$, alors que les desmochores ainsi que les pogonochores sont, chacun, sur une seule espèce.

Les données écologiques des 37 plantes d'hygiène intime de la femme révèlent 9 types de distribution phytogéographique (Figure 14).

La Figure 14 indique que la distribution phytogéographique du type Afro-tropicale concerne la majorité de plantes, soit 35\% d'entre elles. Les types de distribution afroaméricaine, congolaise et cosmopolite ne sont représentés que par une espèce, soit $3 \%$.

$\mathrm{Du}$ point de vue de l'habitat des espèces inventoriées, les 37 espèces se retrouvent dans 6 types de biotopes différents (Figure 15).

Les espèces de savane sont prédominantes $(51 \%)$ parmi les plantes d'hygiène intime de la femme à Kinshasa. Elles sont successivement suivies des espèces de forêt secondaire $(19 \%)$ puis primaire (14\%).

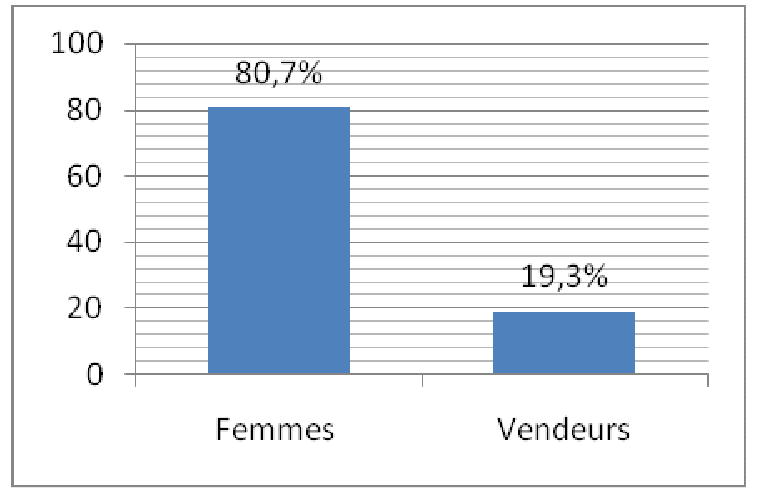

Figure 2 : Répartition des personnes interrogées.

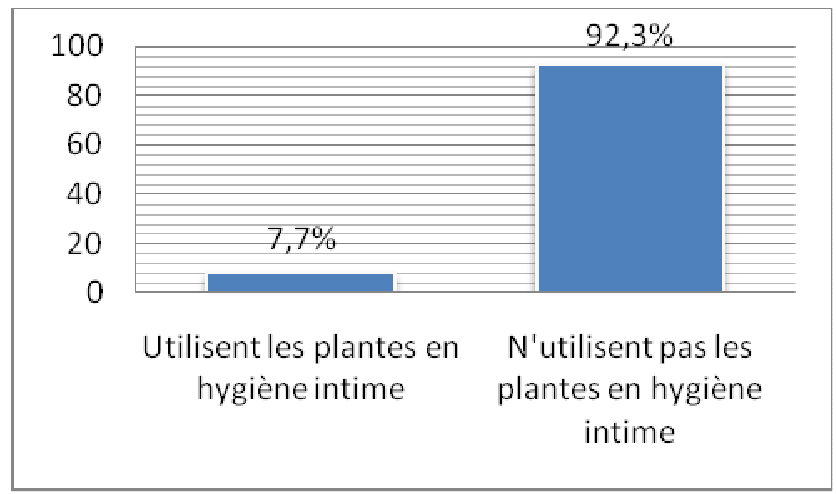

Figure 3: Répartition des personnes interrogées en fonction de l'utilisation des plantes. 
Tableau 1 : Liste des plantes inventoriées auprès des femmes et vendeurs.

\begin{tabular}{|c|c|c|c|c|c|}
\hline Espèces & Nom commun & $\mathbf{F}$ & Partie utilisée & But de l'usage & Famille \\
\hline Abrus precatorius $^{a}$ Li, Phgr, Nano, Bal, AT, Sav. & Elengielengi & 63 & Feuille & $(1),(2),(9)$ & Fabaceae \\
\hline Alchornea cordifolia ${ }^{a}$ arb, Mcph, Méso, Sarco, AT, FS. & Mbunzimbunzi & 79 & Feuille & $(1),(2),(10)$ & Euphorbiaceae \\
\hline Anchomanes difformis ${ }^{b} \mathrm{Hv}$, Gtu, Méga, Scl, GCZ, Jach. & Bikwabimbidi & 1 & Rhizome & (11) & Araceae \\
\hline Biophytum helenae ${ }^{b} \mathrm{Ha}, \mathrm{Thd}, \mathrm{Nano}$, Sarco, Paléo, Sav. & Kanganzo & 6 & Feuille & $(1),(5),(9)$ & Oxalidaceae \\
\hline Bridelia ferruginea $^{a}$ arb, Mcph, Méso, Sarco, GCZ, Sav. & Kimwindu & 30 & Ecorce de tronc & $(1),(3),(10)$ & Phyllanthaceae \\
\hline Oncoba welwitschiic arb, Mcph, Méso, Sarco, GC, FS. & Kisani & 35 & Feuille & $(1),(2),(10)$ & Salicaceae \\
\hline Cathornium altissimum ${ }^{b}$ A, Msph, Micro, Ptéro, GC, FP. & Sumbila & 26 & Ecorce racine & (3). & Fabaceae \\
\hline Chenopodium ambrosioides ${ }^{b} \mathrm{Ha}$, Thd, Nano, Scl,Cosm, Sav. & Mansudinsimba & 36 & Feuille & $(1),(3),(8)$ & Amaranthaceae \\
\hline Cissus aralioides ${ }^{c} \mathrm{Li}, \mathrm{Phgr}, \mathrm{Méso}, \mathrm{Sarco}, \mathrm{AT}, \mathrm{FS}$. & Djila dia mbuji & 3 & Feuille & (12) & Vitaceae \\
\hline Citrus limon ${ }^{a}$ arb, Msph, Micro, Sarco, Pan, Sav. & Monokoyangando & 75 & Fruit & $(1),(3),(10)$ & Rutaceae \\
\hline Cleome rutidosperma ${ }^{b}$ Ha, Thd, Micro, Sarco, G, Rud. & Kibata & 1 & Feuille & (1) & Cleomaceae \\
\hline Crossopteryx febrifuga $a^{a}$ arb, Mcph, Nano, Ptéro, G, Sav. & MapelayaZamba & 55 & Feuille & $(1),(3),(7),(10)$ & Rubiaceae \\
\hline Cyperus articulatus $^{b}$ arb, Mcph, Micro, Scl, AT, Sav. & Nsakunsaku & 3 & Nodules & $(1),(8)$ & Cyperaceae \\
\hline Dewevrea bilabiata ${ }^{b} \mathrm{Hv}, \mathrm{Gzh}, \mathrm{Méso}, \mathrm{Sarco}, \mathrm{GC}, \mathrm{Sav}$. & Kuba kuba & 32 & Follicule & $(1),(3)$ & Fabaceae \\
\hline Fadogia triphylla ${ }^{b}$ Li, Phgr, Nano, Bal, AT, Sav. & Longo longo & 20 & Feuille & $(1),(5)$. & Rubiaceae \\
\hline Gossypium barbadense ${ }^{b} \mathrm{Hv}$, Gzh, Méso, Bal, AT, Sav. & Coton & 18 & Feuille & $(1),(3)$ & Malvaceae \\
\hline 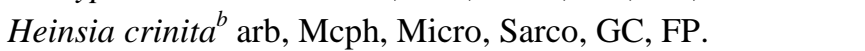 & Siamuna & 18 & Feuille & $(1),(3),(5),(8)$ & Rubiaceae \\
\hline Hibiscus mechowii ${ }^{a}$ S/arb, Nph, Méso, Desm Paléo Sav. & Ngaingaizamba & 67 & Feuille & $(1),(9),(13)$ & Malvaceae \\
\hline Hymenocardi aacida ${ }^{b}$ S/arb, Nph, Méso, Desmo, Paléo, Sav. & Mpete & 30 & Graine & $(1),(10)$ & Phyllanthaceae \\
\hline Lannea antiscorbutica ${ }^{b}$ arb, Mcph, Nano, Ptéro, AT, Sav. & Mukombo & 22 & Racine & $(1),(8),(10)$ & Anacardiaceae \\
\hline Lycopersicon esculentum $^{c}$ A, Msph, Méso, Sarco, GC, FS. & Tomate & 45 & Feuille & $(1),(3),(10)$ & Solanaceae \\
\hline Mangifera indica ${ }^{a} \mathrm{Ha}, \mathrm{Thp}, \mathrm{Micro}$, Sarco, AA, Cult. & Manga & 68 & Feuille ou Ecorce de tronc & $(1),(3),(8)$ & Anacardiaceae \\
\hline Manotes expansa ${ }^{b}$ A,Msph, Méso,Sarco, Pan, FS. & Diladila & 27 & Feuille & $(1),(3)$ & Connaraceae \\
\hline
\end{tabular}


N.O.KABENA et al. / Int. J. Biol. Chem. Sci. 8(6): 2626-2642, 2014

\begin{tabular}{|c|c|c|c|c|c|}
\hline Maprounea africana $^{b}$ Li, Phgr, Micro, Sarco, C, FS. & Selesele & 28 & Feuille & (4) & Euphorbiaceae \\
\hline Monodora myristica ${ }^{b}$ arb, Msph, Micro, Bal, AT, Sav. & Mpeya & 29 & Follicule & $(1),(3),(8)$ & Annonaceae \\
\hline Morinda lucida ${ }^{b}$ A, Msph, Micro, Sarco, GC, FP. & Nsiki & 31 & Feuille & $(1),(3)$ & Rubiaceae \\
\hline Ocimum basilicum $^{c}$ ss/arb, Msph, Méso, Scl, GC, FP. & Mazulu & 15 & Feuille & $(3),(10)$. & Lamiaceae \\
\hline Ocimum gratissimum $^{a}$ Ha, Thd, Micro, Scl, Pan,Sav. & Lumbalumba & 41 & Feuille & $(3),(10)$. & Lamiaceae \\
\hline Pentadiplandra brazzeana ${ }^{b}$ arb, Chd, Micro, Sarco, Paléo, Sav. & Kengekiasa & 2 & Feuille & $(1),(3)$ & Pentadiplandraceae \\
\hline Ricinus communis ${ }^{c}$ Li, Phgr, Micro, Bal, GC, FS. & Mumona & 1 & Feuille & $(1),(3),(6)$ & Euphorbiaceae \\
\hline Sarcocephalus latifolius ${ }^{b}$ arb, Mcph, Méso, Sarco, AT, Cult. & Nlolo & 33 & Ecorce de racine & $(1),(3),(9)$ & Rubiaceae \\
\hline Sclerocroton cornutus ${ }^{b}$ arb, Mcph, Méso, Sarco, AT, Sav. & Kitiki & 15 & Feuille & $(5)$ & Euphorbiaceae \\
\hline Securidaca longipendunculata ${ }^{b}$ arb, Mcph, Méso, Bal, GC, Jach. & Nsunda & 23 & Feuille & $(1),(3)$ & Polygalaceae \\
\hline Tetradenia riparia ${ }^{a}$ aarb, Mcph, Micro, Ptéro, AT, FP. & Mutuzo & 37 & Feuille & $(1),(7)$. & Lamiaceae \\
\hline Vigna unguiculata $^{b}$ Ha, Thd, Méso, Sarco, AT, Cult. & Mbuengi & 24 & Ecorce racine & $(1),(3),(8)$. & Fabaceae \\
\hline Vitex madiensis $^{b}$ arb, Mcph, Méso, Sarco, AT, Sav. & Kifilu & 27 & Feuille & $(1),(6)$ & Lamiaceae \\
\hline Zingiber officinale $^{a} \mathrm{Hv}$, Gzh, Macro, Sarco, G, Sav. & Tangawisi & 70 & Rhizome & $(1),(10)$ & Zingiberaceae \\
\hline
\end{tabular}

$\mathrm{F}:$ Fréquence $\% ;{ }^{a}$ : citées à la fois par les femmes et les vendeurs $;^{b}:$ citées uniquement par les vendeurs $;^{c}:$ citées seulement par les femmes interrogées ; (1) : Rétrécir le canal vaginal ; $(6):$ Favoriser l'involution de l'utérus ab libitum ; (2) : Restaurer la virginité ; (7): Soigner les infections hémorroïdaires ;(3) : Réduire les leucorrhées ; (8): Arrêter les lochies ab libitum ; (4) : Traiter le cancer utérin ; (9)

: Induire la succulence au vagin ; (5): Soigner les infections caractérisées par un prurit ; (10): Tonifier les muscles vaginaux ayant relâché après menstruation ou accouchement ; (11) Soigner

l'infertilité féminine; (12): Etirer les petites lèvres; (13) : Susciter le désir sexuel. 


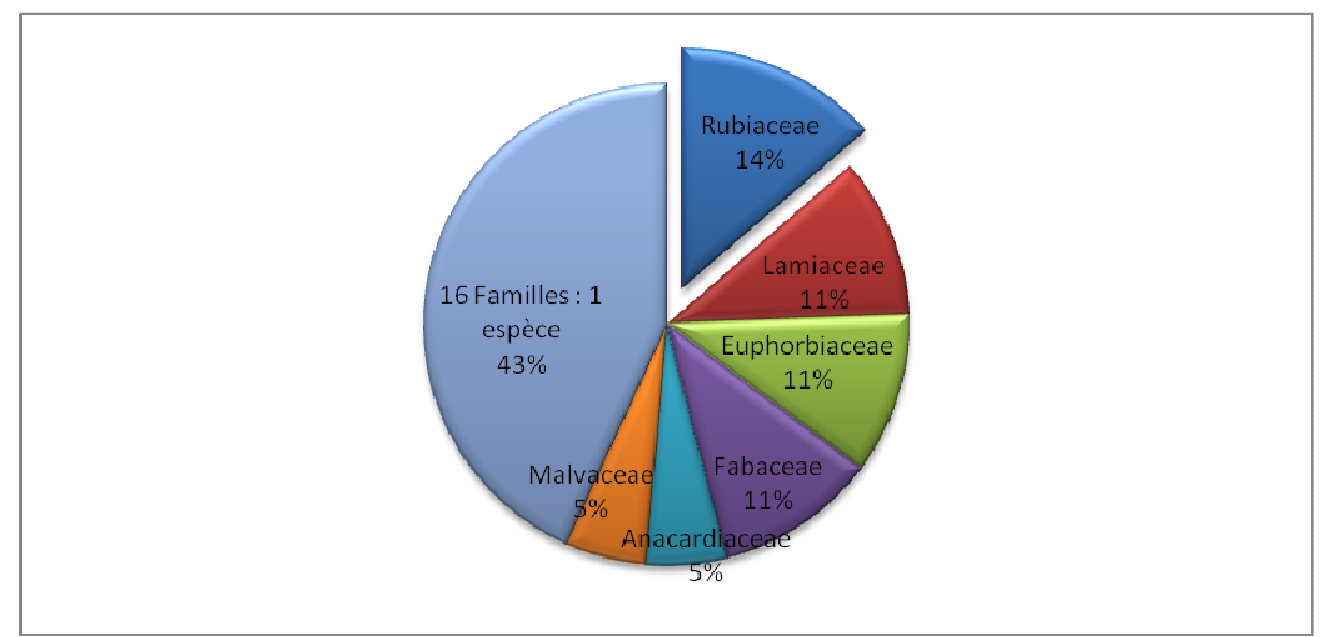

Figure 4 : Importance relative de familles botaniques.

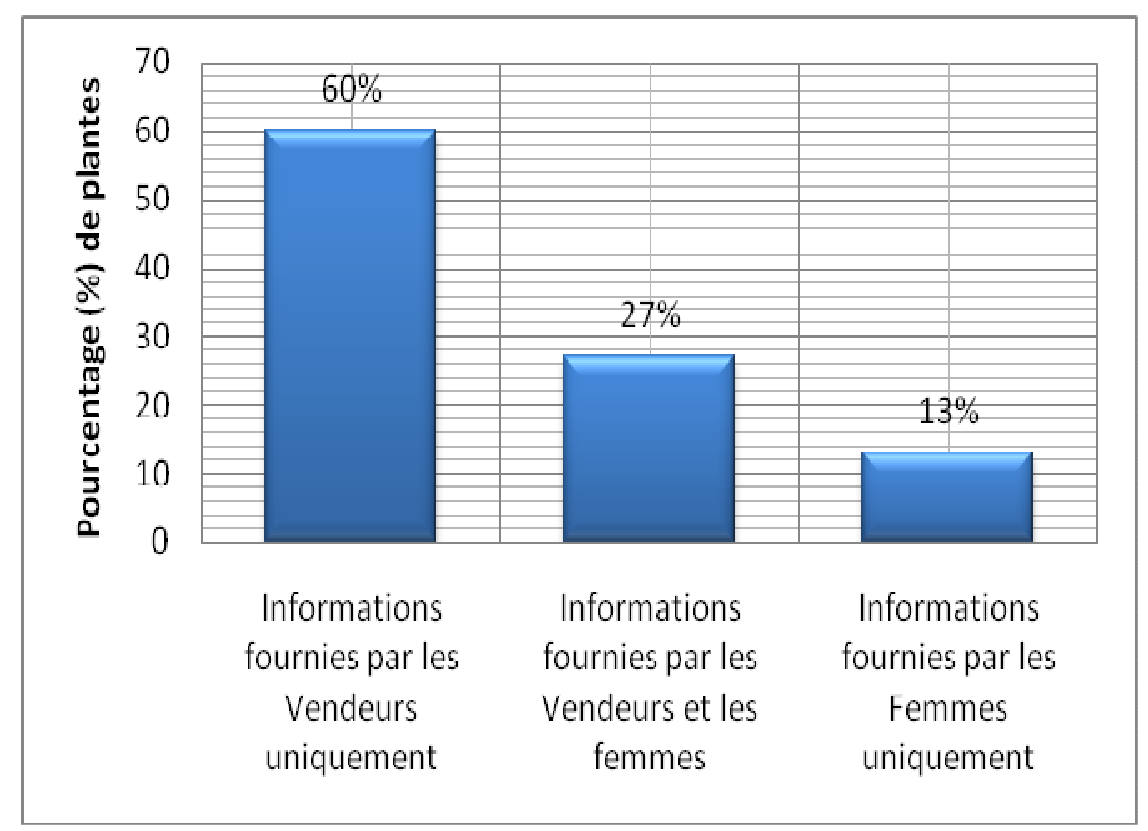

Figure 5: Répartition d'informations sur les plantes en fonction des détenteurs du savoir traditionnel. 


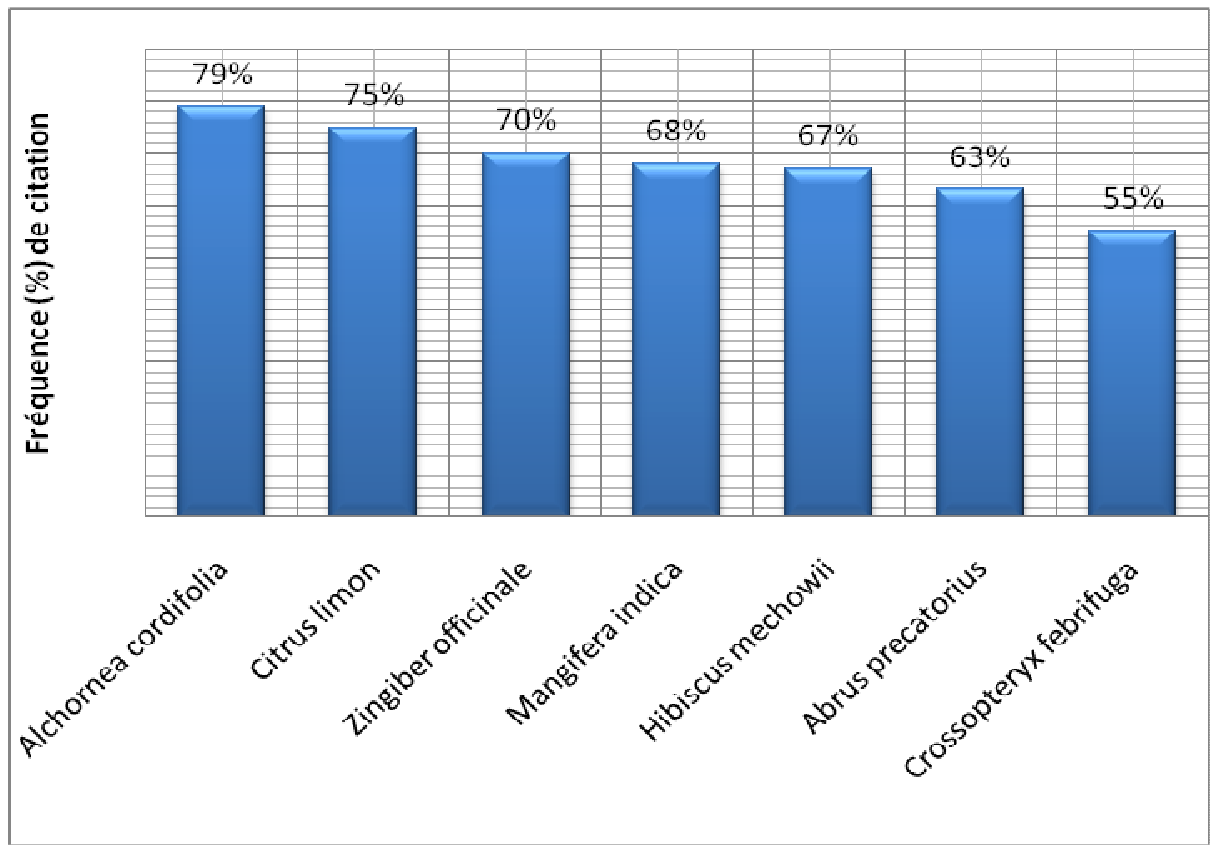

Figure 6: Proportions de plantes les plus citées par les personnes interrogées.

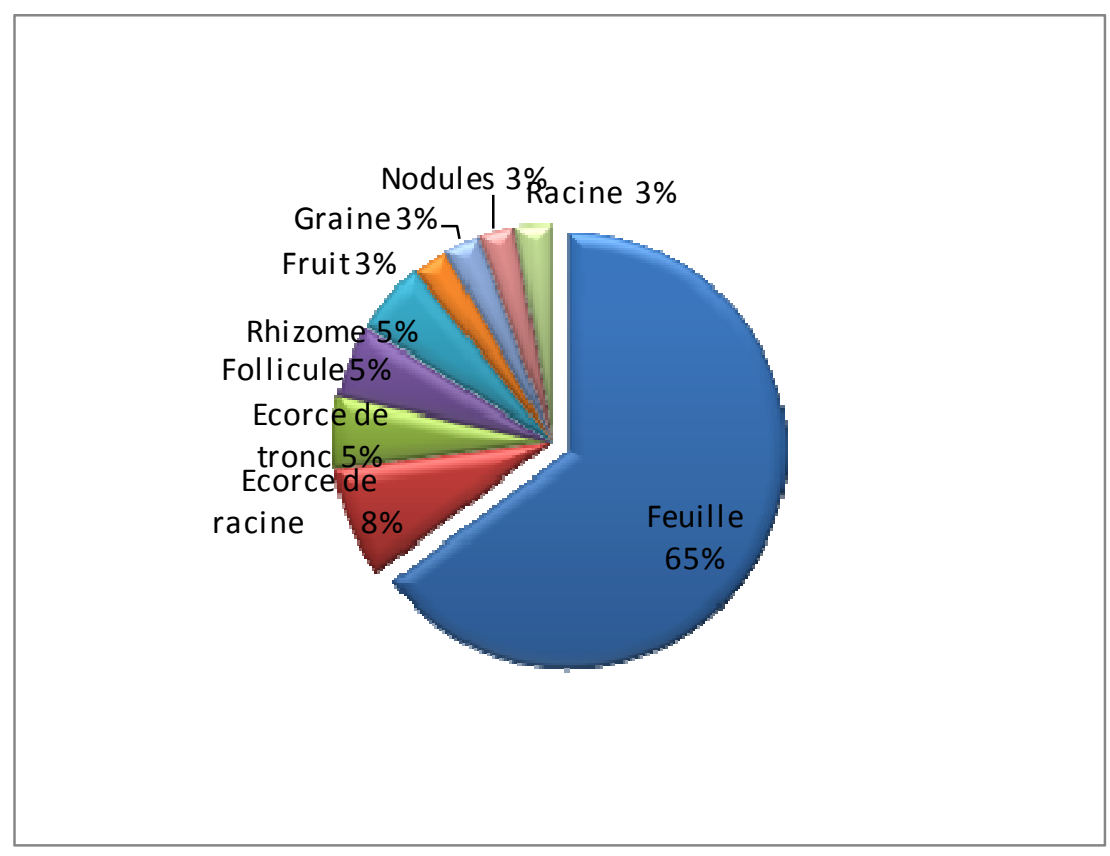

Figure 7: Fréquence des parties de plantes utilisées. 


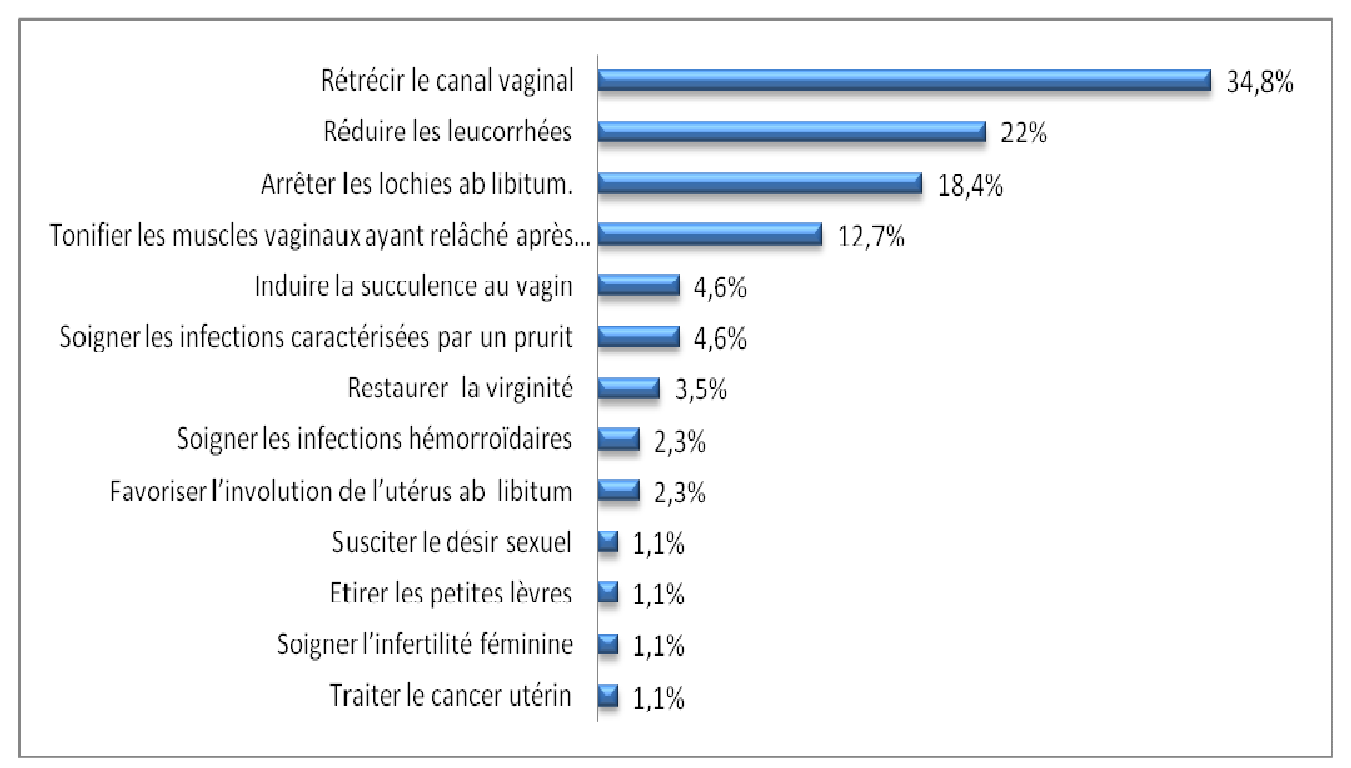

Figure 8: Buts d'usage de plantes en soins intimes et leurs fréquences de citation.

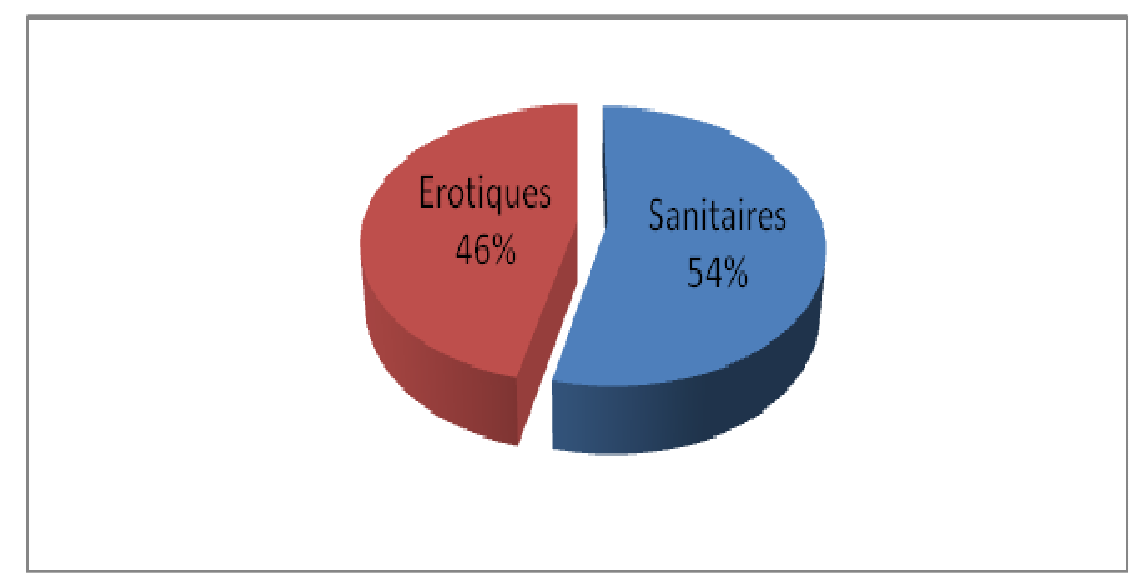

Figure 9: Répartition des buts d'usage des plantes en hygiène intime chez la femme. 


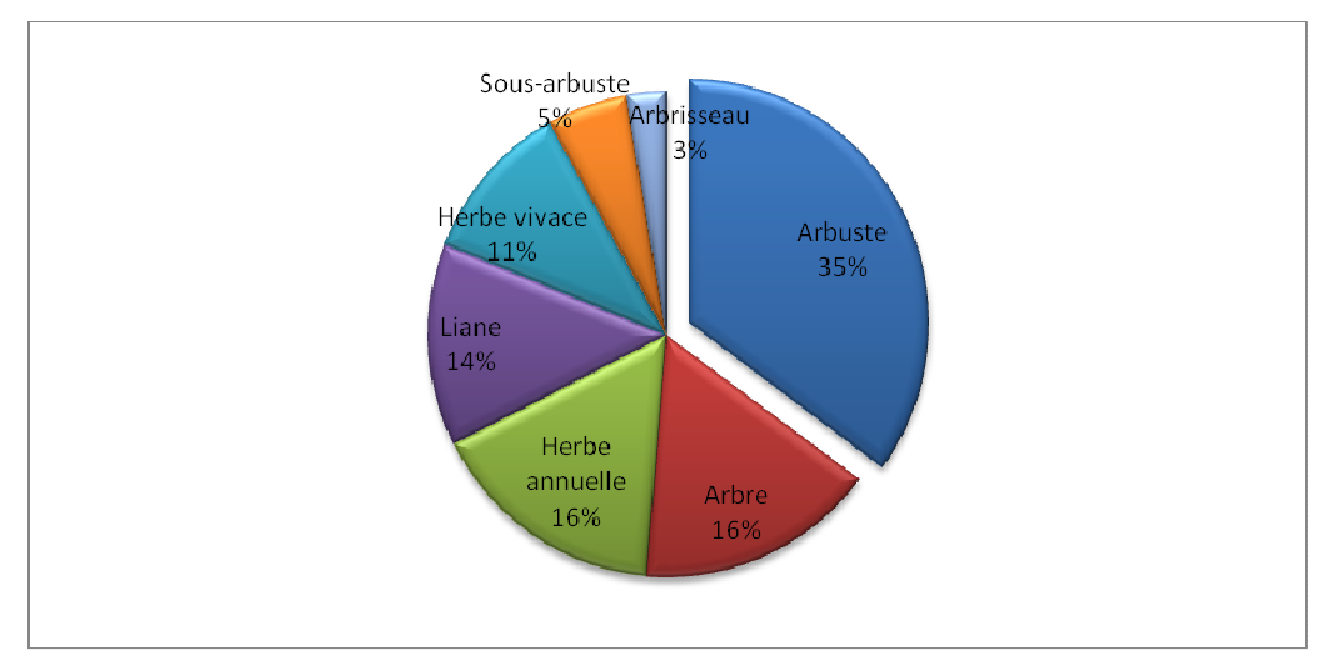

Figure 10: Fréquence relative des types morphologiques d'espèces inventoriées.

Microphanérophyte

- Mésophanérophyte

๑ Phanérophyte grimpant

Thérophyte dressé

⿴囗Géophyte rhizomateux

Mésophanerophyte

Nanophanérophyte

⿴囗十 Chaméphytedressé

$\square$ Géophyte tubéreux

⿶ Thérophyte prostré

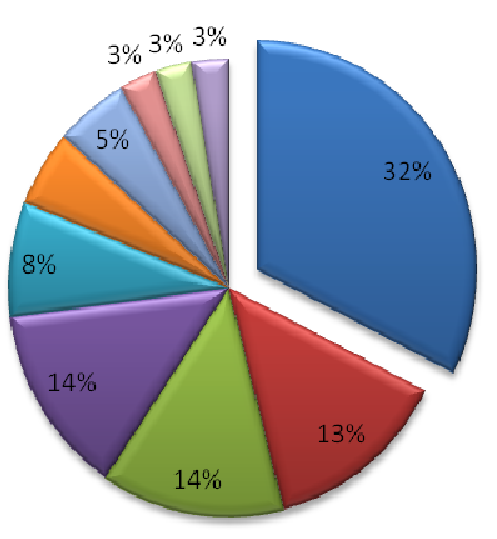

Figure 11: Diversité et proportions de types biologiques d'espèces d'hygiène intime. 


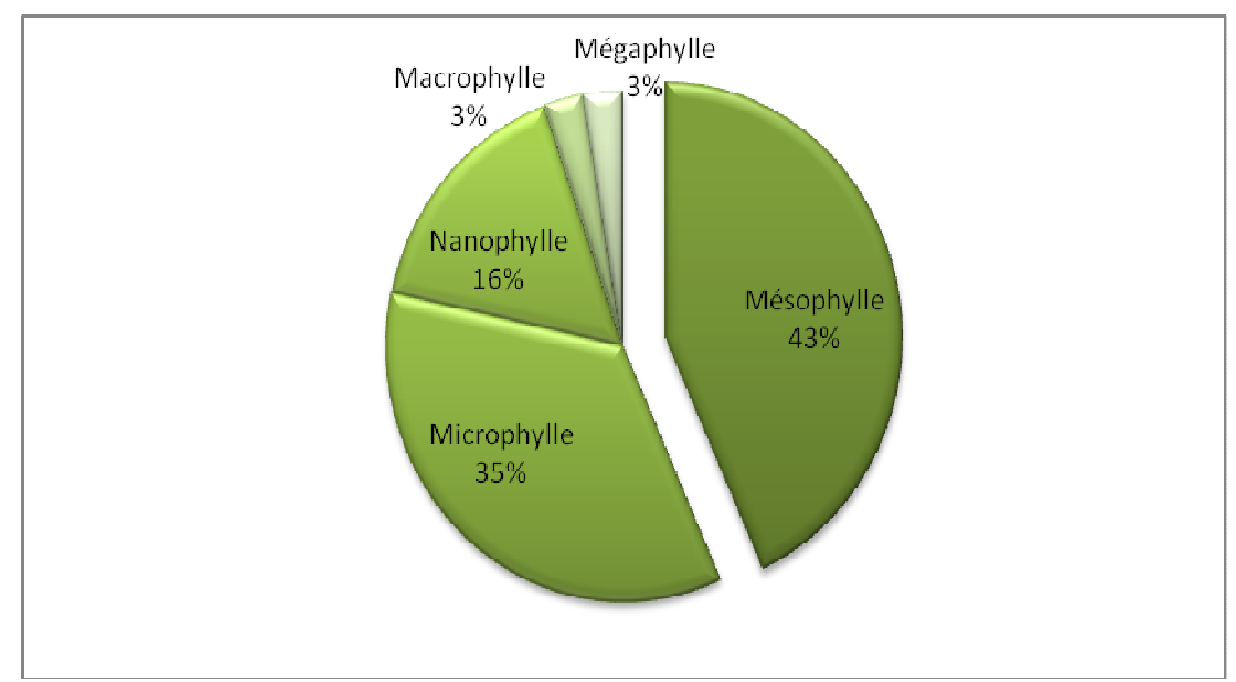

Figure 12: Distribution de types foliaires chez les plantes inventoriées.

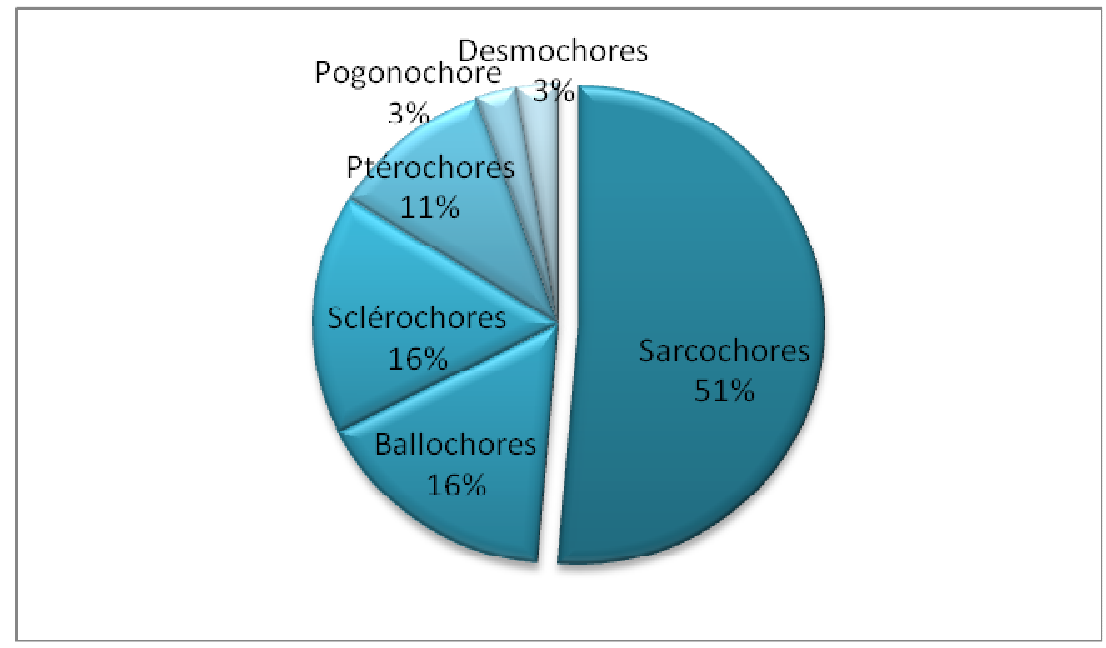

Figure 13: Répartition des types de diaspores de plantes d'hygiène intime. 


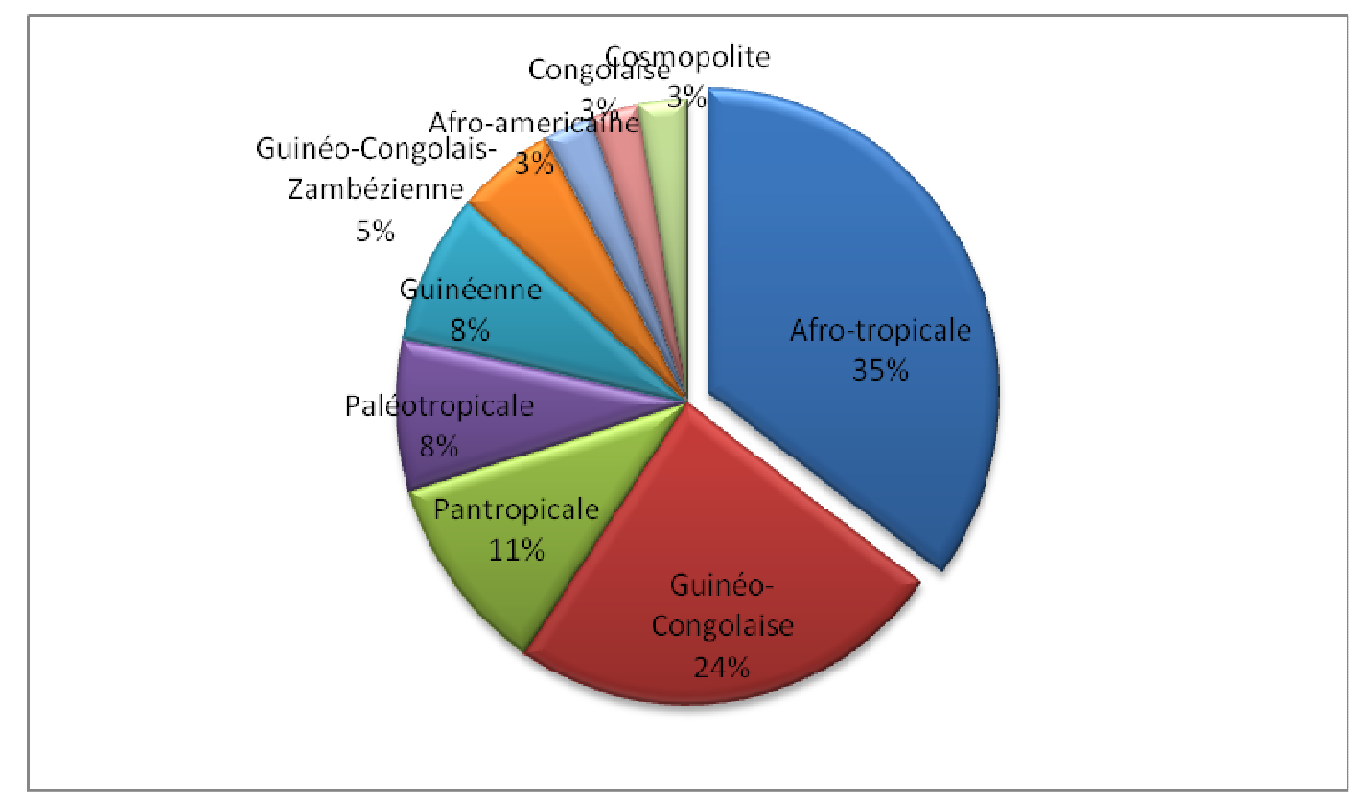

Figure 14: Diversité de plantes en fonction de distribution phytogéographique.

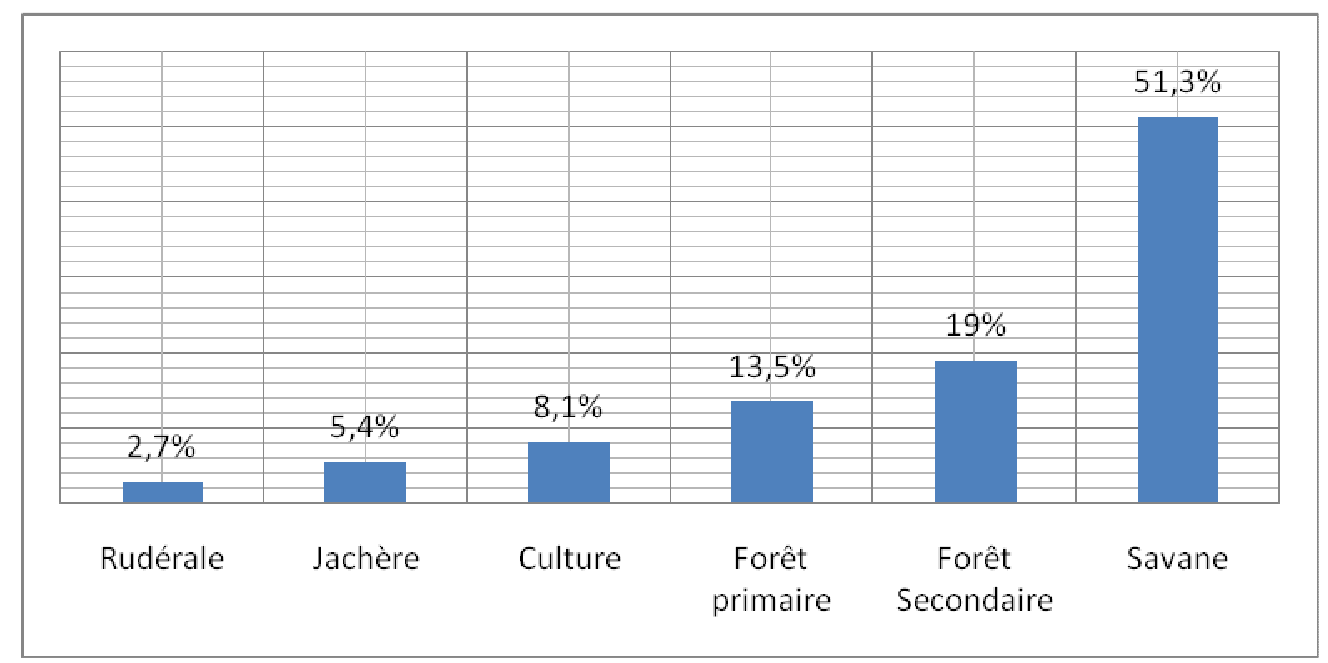

Figure 15 : Types de biotope et fréquence de répartition d'espèces. 


\section{DISCUSSION}

La pratique d'hygiène chez les femmes est très bien documentée. En effet, quelques travaux présentent différentes pratiques d'insertion d'objets dans le tractus vaginal. Ces objets peuvent être des organes de plantes ou les produits commercialisés (Scorgie et al., 2009 ; Hilber et al., 2010). Cependant, les plantes d'hygiène intime de la femme ont rarement fait l'objet de travaux de recherche. Cette étude est parmi les premiers travaux consacrés à ce sujet en République Démocratique du Congo.

La famille Rubiaceaea est majoritairement représentée, au cours de cette étude ethnobotanique. La plupart des plantes inventoriées sont citées dans la littérature comme douées de diverses propriétés pharmacologiques intéressantes. Plus de 60 espèces des Rubiaceae sont utilisées pour plus de 70 indications médicinales incluant le paludisme, l'hépatite, l'eczéma, l'œdème, la toux, l'hypertension, le diabète et la faiblesse sexuelle. Des activités anti-malarique, antimicrobienne, antihypertenseur, antidiabétique, anti-oxydative et anti-inflammatoire ont déjà été démontrées avec plusieurs espèces de cette famille (Kourou et al., 2011). Toutefois, selon plusieurs travaux, l'insertion vaginale des produits est généralement associée à l'augmentation de risques sanitaires notamment l'inflammation de la paroi vaginale, la vaginose bactérienne, la transmission des IST et VIH, la perturbation de la flore vaginale (Brown et al., 2000; Karim et al., 2005 ; Atashili et al., 2008).

En comparant la liste de 37 plantes d'hygiène intime issue de cette étude avec celle de Brown et al. (1992) qui reprend les plantes utilisées par 21 prostituées de la ville de Kananga en RD Congo, trois plantes d'entre elles sont communes. Il s'agit notamment de: Alchornea cordifolia, Hibiscus mechowii et Ocimum gratissimum. Cependant, par rapport aux travaux de Tinde et al. (2008) seul le genre Maprounea est commun, avec les espèces $M$. guianensis et $M$. africana. Le jus de Citrus limon est le seul produit végétal mentionné pour resserrer le vagin chez les femmes zimbabwéennes (Turner et al., 2010).

Alchornea cordifolia, plante la plus citée, est mainte fois reprises dans la littérature pour d'autres fins thérapeutiques (Ebi, 2001 ; Agbor et al., 2004 ; Umukoro et al., 2010; Mpiana et al., 2007). Certaines espèces végétales reprises comme plantes d'hygiène intime sont également utilisées pour d'autres fins médicinales ou encore comme plantes alimentaires, condimentaires, aromatiques ou textiles. C'est le cas notamment de: Citrus limon, Mangifera indica, Lycopersicon esculentum, Ocimum basilicum, Ocimum gratissimum, Vigna unguiculata et Zingiber officinale. Nos investigations montrent que les femmes utilisent les plantes locales disponibles pour l'usage intime. Le screening chimique ainsi que le $\mathrm{pH}$ des recettes à base des plantes inventoriées ont été récemment rapportés dans la littérature (Kabena et al., 2014).

En ce qui concerne les sujets interviewés, les vendeurs des plantes médicinales constituent une source importante des données sur les plantes médicinales en générale et celles utilisées pour l'hygiène intime des femmes en particulier. Sur l'utilisation de plantes hygiène intime, environ $8 \%$ de femmes seulement déclarent utiliser cette pratique. Ce faible taux des femmes utilisatrices serait le fait que l'enquête se soit déroulée en pleine ville cosmopolite et «modernisée » et que la pratique paraitrait comme dégradante et honteuse.

Cinquante quatre pourcent $(54 \%)$ de plantes sont utilisées pour des raisons sanitaires (hygiénique ou thérapeutique) et quarante six $(46 \%)$ pour des raisons liées à l'acte sexuel. Parmi les buts à atteindre par les utilisatrices, le rétrécissement vaginal, la réduction des pertes vaginales et l'arrêt des lochies $a b$ litum ainsi que la tonification des muscles vaginaux sont en bonne position. Ils 
forment à eux seuls environ $88 \%$ de buts d'usages et renseignent sur la perception de la population en ce qui concerne la qualité du vagin. Chez les femmes de Suriname, les 3 raisons majeures sont également le nettoyage du vagin, son resserrement et le nettoyage de l'utérus après accouchement (Tinde et al., 2008). Le consensus étant que le vagin devrait être propre, serré, ferme et chaud (Hilber et al., 2010).

L'utilisation de la feuille dans la préparation de la plupart des recettes d'hygiène intime, contribuerait à la gestion durable de ces espèces végétales; sa cueillette étant moins dangereuse sur le plan environnemental que celle des parties souterraines (Ngbolua et al., 2013). En outre, nos résultats montrent également que ces taxons végétaux occupent les habitats divers et sont largement distribués dans le monde. Ainsi donc, leur protection devrait être un effort concerté tant au niveau national, sousrégional que régional (Ngbolua et al., 2014).

\section{Conclusion}

L'enquête ethnobotanique a permis de dresser une liste de 37 plantes proposées par les femmes et les vendeurs de plantes médicinales, comme plantes d'hygiène intime de la femme. Leurs différents usages en hygiène intime diffèrent selon les buts à atteindre par les utilisatrices; ce qui donne lieu à un multiple usage pour une même espèce végétale. L'inventaire ethnobotanique a permis de découvrir une richesse culturelle et traditionnelle des plantes d'hygiène intime. Toutefois, ces plantes peuvent être sources des métabolites ayant des propriétés biologiques intéressantes au niveau vaginal.

Ce travail de base, vise à contribuer à une plus grande connaissance des plantes médicinales de Kinshasa et ses environs ainsi qu'à leur valorisation comme sources des nouveaux produits contraceptifs ou des microbicides vaginaux.

\section{REFERENCES}

Agbor GA, Léopold T, Jeanne NY. 2004. The antidiarrhoeal activity of Alchornea cordifolia leaf extract. Phytother Res., 18(11): 873-876.

Atashili J, Poole C, Ndumbe PM, Adimora AA, Smith JS. 2008. Bacterial vaginosis and HIV acquisition: a metaanalysis of published studies. AIDS, 22(12):14931501.

Bohbot JM. 2008. Les sécrétions vaginales. Pelvi-périnéologie Springer, 3(1): 19-24.

Bohbot JM et Lepargneur JP. 2012. La vaginose en 2011: Encore beaucoup d'interrogation. Gynécologie Obstétrique et Fertilité, 40(1): 31-36.

Brown RC, Brown JE, Ayowa OB. 1992. The use and physical effects of intravaginal substances in Zairian women. Sexually Transmitted Diseases, 20(2):96-99.

Brown JE, Brown RC. 2000. Traditional intravaginal practices and the heterosexual transmission of disease: a review. Sex Transmitted Diseases, 27(4):183-187.

Ebi GC. 2001. Antimicrobial activities of Alchornea cordifolia. Fitoterapia, 72(1): 69-72.

FAO. 2009. Deuxième rapport national sur l'état des ressources phytogénétiques pour l'alimentation et l'agriculture. R.D. Congo. Préparé dans le cadre du projet FAOTCPRDC/3104.

Flore d'Afrique centrale (Zaïre-RwandaBurundi). Spermatophytes. 1972-1992. Jardin botanique national de Belgique.

Flore du Congo belge et du Ruanda- Urundi. Spermatophyte. 1948-1960. Bruxelles. Publ. I.N.E.A.C. Vol, I-VII et IX.

Flore du Congo, du Rwanda et du Burundi. Spermatophytes. 1967-1971. Jardin botanique national de Belgique.

Flore du Congo, du Rwanda et du Burundi. Spermatophytes, 1962 et 1963. Bruxelles. Publ. I .N .E.A.C. Vol. VIII, et X. 
Gafos M, Mzimela M, Sukazi S, Pool R, Montgomery C, Elford J. 2010. Intravaginal insertion in KwaZulu-Natal: sexual practices and preferences in the context of microbicide gel use. Culture, Health and Sexuality, 12(8):929-942.

Hilber AM, Hull TH, Preston-Whyte E, Bagnol B, Smit J, Wacharasin C, Widyantoro N, the WHO GSVP Study Group. 2010. A cross cultural study of vaginal practices and sexuality: implications for sexual health. Social Science and Medicine, 70(3):392-400.

Kabena NO, Lukoki LF, Mpiana TP, Ngombe KN, Fatiany RP, Baholy R, Ngbolua KN. 2014. Phytochemical Screening of some medicinal plants traditionally used by African Women in Kinshasa city (DR Congo) for their intimate hygiene and Evaluation of the $\mathrm{pH}$ of derived recipes. Journal of Modern Drug Discovery and Drug Delivery Research. V1I3. DOI: 10.15297/JMDDR.V1I3.04.

Karou SD, Tchacondo T, Ilboudo DP, Simpore J. 2011. Sub-saharan rubiaceae: a review of their traditional uses, phytochemistry and biological activities. Pakistan Journal of Biological Sciences, 14(3):149-169.

Kikufi BA. 2012. La Classification Phylogénétique Aujourd'hui. Ed. Herbarium: Kinshasa.

Moroh JL, Bahi C, Dje K, Loukou YG, Guede-Guina F. 2008. Étude de l'activité antibactérienne de l'extrait acétatique (EAC) de Morinda morindoides (Baker) milne-redheat (rubiaceae) sur la croissance in-vitro des souches d'Escherichia coli. Bulletin de la Société Royale des Sciences de Liège, 77:44 - 61.

Mpiana PT, Mudogo V, Tshibangu DST, Ngbolua KN, Shetonde OM, Mangwala PK, Mavakala BK. 2007. In vitro Antisickling Activity of Anthocyanins Extracts of a Congolese Plant: Alchornea cordifolia M.Arg. J. Med. Sci., 7(7): 1182-1186.

Mpiana PT, Ngbolua KN, Mudogo V, Tshibangu DST, Atibu EK. 2012. The potential effectiveness of medicinal plants used for the treatment of Sickle cell Disease in the Democratic Republic of Congo folk medicine: A review. In Progress in Traditional and Folk Herbal Medicine. Daya Publishing House: Delhi; 1-11.

Ngbolua KN, Benamambote BM, Mpiana PT, Muanda DM, Ekutsu E, Tshibangu DST, Gbolo BZ, Muanyishay CL, Basosila NB, Bongo GN, Baholy R. 2013. Ethnobotanical survey and Ecological Study of some Medicinal Plants species traditionally used in the District of BasFleuve (Bas-Congo Province, Democratic Republic of Congo). Research Journal of Chemistry, 1(2):1-10.

Ngbolua KN, Rafatro Herintsoa, Rakotoarimanana Hajatiana, Ratsimamanga Urverg Suzanne, Mudogo V, Mpiana PT, Tshibangu DST. 2011. Pharmacological screening of some traditionally-used anti-malarial plants from the Democratic Republic of Congo compared to its ecological taxonomic equivalence in Madagascar. Int. J. Biol. Chem. Sci., 5(5): 1797-1804.

Ngbolua KN, Mpiana PT, Mudogo V, Ngombe NK, Tshibangu DST, Ekutsu E, Kabena ON, Gbolo BZ, Muanyishay L. 2014. Ethno-pharmacological survey and Floristical study of some Medicinal Plants traditionally used to treat infectious and parasitic pathologies in the Democratic Republic of Congo. International Journal of Medicinal Plants. Photon, 106: 454-467.

Ngombe KN. 2010. Contribution à l'étude phytochimique et détermination de quelques activités biologiques d'Anthocleista schweinfurtii (Gilg) 
Gentianaceae. Thèse de $\mathrm{PhD}$, Université de Kinshasa, Kinshasa, p. 227.

Reid G. 2002. Probiotics for urogenital health. Nutr. Clin. Care, 5(1):3-8.

Rousseau MV. 2004. Evaluation d'oligosaccharides à effet prébiotique visà-vis de la microflore vaginale. Thèse de $\mathrm{PhD}$, Institut National des Sciences Appliquées de Toulouse, Toulouse, p. 185.

Saint Moulin L, Kalombo TJL. 2005. Atlas de l'Organisation Administrative de la République Démocratique du Congo. CEPAS: Kinshasa.

Scorgie F, Kunene B, Smit JA, Manzini N, Chersich MF and Preston-Whyte EM. 2009. In search of sexual pleasure and fidelity: Vaginal practices in KwaZuluNatal, South Africa. Culture, Health and Sexuality, 11(3):267-83.
Tinde VA, Sanned K, Daphne KS, Joelaika BR, Sofie R. 2008. Dry sex in Suriname. Journal of Ethnopharmacology, 116(1):84-88.

Turner AN, Morrison CS, Munjoma MW, Moyo P, Chipato T, Wijgert JH. 2010. Vaginal Practices of HIV-Negative Zimbabwean Women. Infectious Diseases in Obstetrics and Gynecology, Article ID 387671, 7p.

Umukoro S, Aladeokin AC. 2010. Evaluation of the anti-stress and anticonvulsant activities of leaf extract of Alchornea cordifolia in mice. J. Ethnopharmacol, 127(3):768-770.

WHO. 2002. Médecine traditionnelle. http:www.who.int/mediacentre/factsheets/ 2003/fs134/fr/ (accès décembre, 2011). 